\title{
Coagulase-Positive Staphylococcus
}

National Cancer Institute

\section{Source}

National Cancer Institute. Coagulase-Positive Staphylococcus. NCI Thesaurus. Code C62585.

A subtype of Staphylococcus characterized by an ability to produce free plasma coagulase. 